\title{
Adjustable Solutions of Doubly Coprime Matrix Fraction Descriptions
}

\author{
Hung-Chou Chen \\ Department of Electrical Engineering \\ National Taiwan University \\ Taipei, Taiwan, 10619
}

\author{
Fan-Ren Chang \\ Department of Electrical Engineering \\ National Taiwan University \\ Taipei, Taiwan, 10619
}

\begin{abstract}
Using the concept of infinite eigenstructure assignment in generalized systems, explicit formulas for calculating the polynomial generalized Bezout identity is proposed. The degree of the polynomial matrix is directly related to the length of the longest infinite eigenvector chain of the associated generalized state-space representation. Hence, the method of infinite eigenstructure assignment can be used to find adjustable-degree solutions of the doubly coprime matrix fraction descriptions.
\end{abstract}

Keywords : Generalized systems, Infinite eigenstructure assignment, Polynomial generalized Bezout identity.

\section{Introduction and Formulation Problem}

The left matrix fraction description of a linear time-invariant system is usually described by $\bar{D}^{-1}(s) \bar{N}(s)$. We suppose that $\bar{D}(s) \in R|s|^{m \times m}$ and $\bar{N}(s) \in R[s]^{m \times r}$ are left coprime and $\bar{D}(s)$ is row reduced, where $R[s]^{m \times m}$ and $R[s]^{m \times r}$ indicate the set of all $m \times m$ and $m \times r$ real coefficient polynomial matrices respectively. A simple approach is proposed here to determine six polynomial matrices $\bar{X}(s), \bar{Y}(s), D(s), N(s), X(s)$, and $Y(s)$ of appropriate dimensions to satisfy

$$
\left[\begin{array}{cc}
\bar{D}(s) & \bar{N}(s) \\
-Y(s) & X(s)
\end{array}\right]\left[\begin{array}{cc}
\bar{X}(s) & -N(s) \\
\bar{Y}(s) & D(s)
\end{array}\right]=\left[\begin{array}{cc}
I & 0 \\
0 & I
\end{array}\right]
$$

Throughout the development of this paper, we first assume $m \leq r$ and $\left\{\delta_{r i} \bar{N}(s) \leq \delta_{r i} \bar{D}(s)\right.$, for $i=$ $1,2, \ldots, m\}$, where $\left.\delta_{r i} \mid.\right]$ stands for the ith row 0-7803-1800-5/94/\$3.00 (C) 1994 IEEE degree. Note that the assumptions of $m \leq r$ can be released in the subsequent development. Equation (1) usually called as the polynomial generalized Bezout identity, which plays a key role in the synthesis and analysis of compensators for linear timeinvariant systems in frequency domain $[2,8,9]$. The computational algorithms for solving the equation can be found in the literature, such as linear algebraic methods in $\{12\}$, and the state-space methods in $[3,4,6]$. However, the adjustable-degree solutions in (1) have rarely been investigated in previous researches.

While the bulk of published papers have been focused on solving the polynomial generalized Bezout identity, the use of infinite eigenstructure assignment in generalized systems to attack this subject is still lacking. The degree of a polynomial matrix ( which is defined as the greatest degree of the polynomials appearing as entries in the polynomial matrix $(10)$ ) is directly related to the length of the longest infinite eigenvector chain. Thus, if we can assign the infinite eigenstructure under the state feedback gain selection, then the adjustable-degree solutions in (1) could be found. In the following, we describe the infinite eigenstructure assignment method for controllable generalized systems first, which is simpler than that of $[5]$.

\section{Infinite Eigenstructure Assignment}

We consider a controllable time-invariant generalized system [13] of the form:

$$
E \dot{x}(t)=A x(t)+B u(t),
$$

where $x(t) \in R^{n}$ is the state vector, $u(t) \in R^{r}$ is the input vector, $E, A$ and $B$ are real constant matrices of appropriate dimensions. We assume that 
$E$ is singular and its rank is $g$ which is less than $\mathbf{n}$. Furthermore, we assume that $s E-A$ is a regular pencil (i.e. $|s E-A| \not \equiv 0$ ) such that system (2) is solvable.

Our purpose is to find a state feedback gain matrix $K$ such that the closed-loop system

$$
E \dot{x}(t)=A_{c} x(t), \text { where } A_{c} \triangleq A+B K
$$

contains infinite eigenvalues only. Let algebraic and geometric multiplicities of infinite eigenvalues are $n$ and $\eta$ respectively, then $\eta=$ nullity $E=n-g$. We denote $v_{\infty j}^{(0)}$ be the rank 0 infinite eigenvectors of (3), then $E v_{\infty j}^{(0)}=0, \quad j=1,2, \cdots, \eta$. Also, denote $v_{\infty j}^{(k)}$ be the rank $k$ infinite eigenvectors, then $E v_{\infty j}^{(k)}=$ $A_{c} v_{\infty j}^{(k-1)}, \quad k=1,2, \cdots,\left(p_{\infty j}-1\right)$. Since (3) has $n$ infinite eigenvalues, we have $n\left(=\sum_{j=1}^{n} p_{\infty j}\right)$ linearly independent infinite eigenvectors $v_{\infty j}^{(k)}[11]$ The nonsingular matrix $V_{\infty}$ is constructed as follows :

$$
\begin{gathered}
V_{\infty} \triangleq\left[V_{\infty 1}, V_{\infty 2}, \cdots, V_{\infty j}, \cdots, V_{\infty \eta}\right], \\
V_{\infty j} \triangleq\left[v_{\infty j}^{(0)}, \cdots, v_{\infty j}^{(k)}, \cdots v_{\infty j}^{\left(p_{\infty j}-1\right)}\right],
\end{gathered}
$$

where $j=1,2, \cdots, \eta$ and $k=0,1,2, \cdots,\left(p_{\infty j}-1\right)$. Let us set

$$
f_{\infty j}^{(k)}=K v_{\infty j}^{(k)}
$$

then $f_{\infty j}^{(k)} \in R^{r}$ will help us to find $K$ later. Using the infinite eigenstructure proposed in [11], we can obtain

$$
\begin{gathered}
E v_{\infty j}^{(k)}=A v_{\infty j}^{(k-1)}+B K v_{\infty j}^{(k-1)}=A v_{\infty j}^{(k-1)}+B f_{\infty j}^{(k-1)}, \\
\text { for } k=1,2, \cdots, p_{\infty j}-1, \quad j=1,2, \cdots, \eta .
\end{gathered}
$$

Note that $\left[\begin{array}{lll}E & \vdots & -B\end{array}\right]$ is full row rank, since (2) is controllable. Also, $\left.v_{\infty j}^{(k)}\right|_{k=0}$ being in the null space of $E$, those $v_{\infty j}^{(k)}$ and $f_{\infty j}^{(k-1)}$ can be computed interactively as

$$
\begin{gathered}
{\left[\begin{array}{c}
v_{\infty j}^{(k)} \\
f_{\infty j}^{(k-1)}
\end{array}\right]=\left[\begin{array}{lll}
E & \vdots & -B
\end{array}\right]^{+} A v_{\infty j}^{(k-1)},} \\
\text { for } k=1,2, \cdots, p_{\infty j}-1
\end{gathered}
$$

where $(\cdot)^{+}$represents the matrix generalized inverse of the designated matrix (.). From (7), $f_{\infty j}^{(0)}, f_{\infty j}^{(1)}, \cdots, f_{\infty j}^{\left(p_{\infty},-2\right)}$ can be solved. The selection of $f_{\infty j}^{\left(p_{\infty} ;-1\right)}$ should satisfy the requirement listed in Remark 1. Then we can construct

$$
\begin{gathered}
F_{\infty} \triangleq\left[F_{\infty 1}, F_{\infty 2}, \cdots, F_{\infty j}, \cdots, F_{\infty \eta}\right], \\
F_{\infty j} \triangleq\left[f_{\infty j}^{(0)}, f_{\infty j}^{(1)}, \cdots, f_{\infty j}^{(k)}, \cdots, f_{\infty j}^{\left(p_{\infty j}-1\right)}\right],
\end{gathered}
$$

where $j=1,2, \cdots, \eta$ and $k=0,1,2, \cdots,\left(p_{\infty j}-1\right)$. From (4), (5) and (8), we have $K V_{\infty}=F_{\infty}$, the feedback gain $K$ then becomes

$$
K=F_{\infty} V_{\infty}^{-1} \text {. }
$$

The columns of $V_{\infty}, v_{\infty j}^{(k)}$, are infinite eigenvectors of the closed-loop system, hence they are linearly independent. When (7) is used to create $V_{\infty}$ and $F_{\infty},\left\{v_{\infty 1}^{(0)}, v_{\infty 2}^{(0)}, \cdots, v_{\infty \pi}^{(0)}\right\}$ should be a basis of the null space of $E$. Also, different approaches to find $v_{\infty j}^{(k)}$ may cause the different combinations of chain lengths. For example, we can search the crate by rows along the procedure tabulated in Fig 1. (Similarly like [8], p 427.) Note that if (2) is controllable then all finite eigenvalues can be assigned to infinite ones [5].

\begin{tabular}{|c|c|c|c|}
\hline \multicolumn{5}{|c|}{ eigenvectors } \\
\hline chain & $v_{\infty 1}^{(k)}$ & $\ldots$ & $v_{\infty \eta}^{(k)}$ \\
\hline rank 0 & $\mathrm{x}$ & $\mathrm{x}$ & $\mathrm{x}$ \\
\hline rank 1 & $\mathrm{x}$ & $\mathrm{x}$ & $\mathrm{x}$ \\
\hline rank 2 & 0 & $\mathrm{x}$ & 0 \\
\hline rank 3 & & 0 & \\
\hline$\vdots$ & & & \\
\hline rank p & & & \\
\hline
\end{tabular}

Fig 1. Sequences of independent infinite eigenvectors examination

\begin{tabular}{|c|c|c|c|}
\hline \multicolumn{4}{|c|}{ eigenvectors premultiplied by $E$} \\
\hline chain & $E v_{\infty)}^{(k)}$ & $\ldots$ & $E v_{\infty \eta}^{(k)}$ \\
\hline rank 1 & $\mathbf{x}$ & $\mathbf{x}$ & $\mathbf{x}$ \\
\hline rank 2 & 0 & $\mathbf{x}$ & 0 \\
\hline rank 3 & & 0 & \\
\hline rank 4 & & & \\
\hline$\vdots$ & & & \\
\hline rank p & & & \\
\hline
\end{tabular}

Fig 2. Sequences of independent vectors examination

Remark 1 : Since $s E-A_{c}$ contains infinite eigenvalues only, $s E-A_{c}$ must be unimodular. The determinant of $s E-A_{c}$ is a nonzero constant and equals to $\left|-A_{c}\right|$. Thus, $A_{c}=A+B K=A+$ $B F_{\infty} V_{\infty}^{-1}$ would be nonsingular [5]. Both $A_{\mathrm{c}}$ and $V_{\infty}$ are nonsingular, their product $A_{c} V_{\infty}$ is nonsingular as well. From (6) and $A_{c}=A+B F_{\infty} V_{\infty}^{-1}$ we see that 
$A_{c} V_{\infty}$

$=\left[A_{c} v_{\infty 1}^{(0)}, \cdots, A_{c} v_{\infty 1}^{\left(p_{\infty 1}-2\right)}, A v_{\infty 1}^{\left(p_{\infty 11}-1\right)}+B f_{\infty 1}^{\left(p_{\infty 1}-1\right)} \vdots \ldots\right.$

$\left.\left.A_{c} v_{\infty \eta}^{(0)}, \cdots, A_{c} v_{\infty \eta}^{\left(p_{\infty} \eta-2\right)}, A v_{\infty \eta}^{\left(p_{\infty}-1\right)}+B f_{\infty \eta}^{\left(p_{\infty \eta}-1\right)}\right\}\right]$

$=\left[E v_{\infty 1}^{(1)}, \cdots, E v_{\infty 1}^{\left(p_{\infty 11}-1\right)}, A v_{\infty 1}^{\left(p_{\infty 11}-1\right)}+B f_{\infty 11}^{\left(p_{\infty}-1\right)} \vdots \ldots \vdots\right.$

$\left.\left.E v_{\infty \eta}^{(1)}, \cdots, E v_{\infty \eta}^{\left(p_{\infty},-1\right)}, A v_{\infty \eta}^{\left(p_{\infty \eta}-1\right)}+B f_{\infty \eta \eta}^{\left(\gamma_{\infty}-1\right)}\right\}\right]$

The requirement of nonsingular $A_{c} V_{\infty}$ is equivalent to the following:

(i) Let $S \triangleq\left\{E v_{\infty 1}^{(1)}, E v_{\infty 11}^{(2)}, \cdots, E v_{\infty 1}^{\left(p_{\infty 11}-1\right)} \vdots \ldots\right.$ : $\left.E v_{\infty \eta}^{(1)}, E v_{\infty \eta}^{(2)}, \cdots, E v_{\infty \eta}^{(p)-1)}\right\}$, then $S$ is an independent set. We should examine the independence of $S$ as shown in Fig. 2 by the similar procedures listed in Fig. 1.

(ii) Let $T \triangleq\left\{A v_{\infty 1}^{\left(p_{\infty 1}-1\right)}+B f_{\infty 1}^{\left(p_{\infty}-1\right\}} \vdots \ldots\right.$ : $\left.\left.A v_{\infty \eta}^{\left(p_{\infty}-1\right)}+B f_{\infty \eta}^{(p \infty \eta}-1\right)\right\}$, then the choices of $f_{\infty j}^{\left(P_{\infty j}-1\right)}$ for $j=1,2, \cdots, \eta$ should satisfy the condition of $[S: T]$ being nonsingular.

As a summary of the previous results, we have the following theorem.

Theorem 1 : Suppose (2) is a controllable generalized system, the closed-loop assignable chains of infinite eigenvectors, $v_{\infty j}^{(k)}$, can be written as

$$
K V_{\infty}=F_{\infty} \text { or } K=F_{\infty} V_{\infty}^{-1}
$$

where $V_{\infty}$ and $F_{\infty}$ are shown in (4) and (8) respectively. $v_{\infty j}^{(k)}$ and $f_{\infty j}^{(k)}$ satisfy

$$
\begin{aligned}
& {\left[\begin{array}{c}
v_{\infty j}^{(k)} \\
f_{\infty j}^{(k-1)}
\end{array}\right]=\left(\left[\begin{array}{lll}
E & \vdots & -B
\end{array}\right]\right)^{+} A v_{\infty j}^{(k-1)},} \\
& k=1,2, \cdots, p_{\infty j}-1, \quad j=1,2, \cdots, \eta,
\end{aligned}
$$

where $E v_{\infty j}^{(0)}=0$, under the conditions of

(i) $V_{\infty}$ is nonsingular.

(ii) $\left[E v_{\infty 1}^{(1)}, E v_{\infty 1}^{(2)}, \cdots, E v_{\infty 1}^{\left(p_{\infty 11}-1\right)}, A v_{\infty 1}^{\left(p_{\infty 1}-1\right)}+\right.$ $B f_{\infty 1}^{\left(p_{\infty 1}-1\right)} \vdots \ldots \vdots E v_{\infty \eta}^{(1)}, E v_{\infty \eta}^{(2)}, \cdots, E v_{\infty \eta}^{\left(p_{\infty n}-1\right)}$, $\left.A v_{\infty \eta \eta}^{\left(D_{n}-1\right)}+B f_{\infty \eta}^{\left(p_{\infty}-1\right)}\right\}$ is nonsingular.

The condition (i) is needed due to the requirement of eigenvectors being independent. The condition (ii) is needed because of the requirement of $A_{c}$ being nonsingular ( See Remark 1). Our approach presented here is more straightforward and requires less computation than that in [5]. The above result also provides a useful approach to solve the polynomial generalized Bezout identity.

\section{Solutions of the Polynomial Bezout Identity}

In this section, we shall introduce an efficient method to realize a time-invariant descriptor system whose input-output relationship is given by the $m \times r$ polynomial matrix fraction description $\bar{D}^{-1}(s) \bar{N}(s)$ with $\delta_{r i} \bar{N}(s) \leq \delta_{r i} \bar{D}(s)$. Let $\alpha_{i}=$ $\delta_{r_{i}} \bar{D}(s)+1$. We first define

$$
E=\left\{\text { block diag }\left[\begin{array}{ccccc}
0 & 1 & 0 & \cdots & 0 \\
0 & 0 & 1 & \cdots & 0 \\
\vdots & \vdots & & \ddots & \vdots \\
0 & 0 & 0 & \cdots & 1 \\
0 & 0 & 0 & \cdots & 0
\end{array}\right]_{\alpha, \times \alpha},\right\}
$$

and

$$
C=\left\{\begin{array}{cccc}
\text { block diag }\left[\begin{array}{ccccc}
1 & 0 & \cdots & 0 & 0
\end{array}\right]_{1 \times \alpha_{1}} \\
i=1,2, \cdots, m
\end{array}\right\}
$$

We set $\bar{V}_{\infty}^{(0)}=C^{t}$ ( the transpose of $C$ ), then

$$
\begin{aligned}
& \bar{v}_{\infty}^{(0)} \\
& =\left[\bar{v}_{\infty 1}^{(0)} \cdots \bar{v}_{\infty i}^{(0)} \cdots \bar{v}_{\infty m}^{(0)}\right]
\end{aligned}
$$

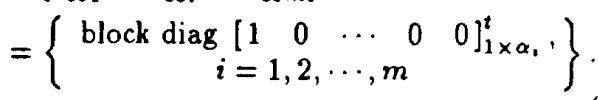

Note that $\left\{\bar{v}_{\infty i}^{(0)}, i=1,2, \cdots, m\right\}$ forms a basis for the nullspace of $E$.

If we set $M(s)=(s E-I)$, then $M(s)$ is a unimodular matrix. $(s E-I)^{-1}$ can be represented as

$$
\begin{aligned}
M(s)^{-1} & =(s E-I)^{-1} \\
& =\left\{I+E s+E^{2} s^{2}+\cdots+E^{q-1} s^{q-1}\right\},
\end{aligned}
$$

where $q \triangleq \max \left\{\alpha_{i}\right.$, for $\left.i=1, \cdots, m\right\}$. This can be further expressed as

$$
\left.\begin{array}{c}
(s E-I)^{-1}= \\
\left\{\text { block diag }\left\{-\left[\begin{array}{cccc}
1 & s & \cdots & s^{\alpha_{1}-1} \\
0 & 1 & \cdots & s^{\alpha_{1}-2} \\
\vdots & \vdots & \ddots & \vdots \\
0 & 0 & \cdots & s \\
0 & 0 & \cdots & 1
\end{array}\right]_{\alpha_{1} \times \alpha_{1}}\right\}\right. \\
i=1,2, \cdots, m
\end{array}\right\}
$$

and

$$
\begin{aligned}
& C(s E-I)^{-1}=
\end{aligned}
$$

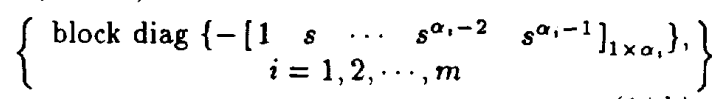


Lemma 1 : Let us consider the $m \times r$ polynomial matrix fraction description $\bar{D}^{-1}(s) \bar{N}(s)$ with $\delta_{r i} \bar{N}(s) \leq \delta_{r i} \bar{D}(s)$. Assume $\bar{D}(s)$ and $\bar{N}(s)$ are left coprime, and $\bar{D}(s)$ is row reduced. Define the highest-row-degree coefficient matrix of $[\bar{D}(s) \vdots \bar{N}(s)]$ be denoted as $\left[D_{h r} \vdots N_{h r}\right]$.

Suppose $m \leq r$ and set $B_{\text {ohr }} \in R^{m \times r}$ be an arbitrary full row rank matrix and $D_{0}=-D_{h r}^{-1}\left(B_{o h r}-\right.$ $N_{h r}$ ). Under these settings, if we choose $L$ and $B$ o satisfying $\bar{D}(s)=I+C M^{-1}(s) L$ where $C, E$ and $M(s)$ are shown in (10), (11) and (13), respectively, and $\bar{N}(s)=D_{0}+C M^{-1}(s)\left(B_{0}+L D_{0}\right)$, then $\bar{D}^{-1}(s) \bar{N}(s)=D_{0}+C W(s)^{-1} B_{0}$, where $A \triangleq I-L C$ and $W(s) \triangleq(s E-A)$. Furthermore, the quadruple $\left\{E, A, B_{o}, C\right\}$ are controllable and observable.

Remark 2 : In Lemma $1, L, B_{0}$ are read directly from the coefficients of $\bar{D}(s)-I$ and $\bar{N}(s)-\bar{D}(s) D_{0}$ since $C(s E-I)^{-1}$ has the form shown in (14.b). Thus, the realization is simplified.

In the previous section, different independent vector searching schemes for determining $K$ will result in different infinite eigenstructures of controllable generalized systems. The computed state feedback gain matrix $K$ will make $A_{c}$ nonsingular and create a unimodular matrix $H(s)$, where $H(s)=s E-A_{\mathrm{c}}=$ $s E-A-B_{0} K$. ( $\left.s E_{\infty}-A_{c \infty}\right)$ is the staircase form of $\left(s E-A_{c}\right)$, this can be obtained under unitary transformations in [1] which is a numerically reliable algorithm.

$$
Q\left(s E-A_{c}\right) Z=\left(s E_{\infty}-A_{c \infty}\right) .
$$

Hence, $\left(s E_{\infty}-A_{c \infty}\right)^{-1}$ can be represented as

$$
\begin{aligned}
& \left(s E_{\infty}-A_{c \infty}\right)^{-1} \\
& =\left(s\left[\left(A_{c \infty}\right)^{-1} E_{\infty}\right]-I\right)^{-1}\left[\left(A_{c \infty}\right)^{-1}\right] \\
& =-\left\{I+\left[\left(A_{c \infty}\right)^{-1} E_{\infty}\right] s+\left[\left(A_{c \infty}\right)^{-1} E_{\infty}\right]^{2} s^{2}+\cdots\right. \\
& \left.+\left[\left(A_{c \infty}\right)^{-1} E_{\infty}\right]^{q_{c}-1} s^{g_{c}-1}\right\}\left[\left(A_{c \infty}\right)^{-1}\right],
\end{aligned}
$$

where $q_{c}=$ nilpotent index $\left[\left(A_{c \infty}\right)^{-1} E_{\infty}\right]$. The highest degree $H^{-1}(s)$ is determined by the length of the longest infinite eigenvector chain denoted as $q_{c}$. With this idea in mind, we will develop adjustable-degree solutions of the polynomial generalized Bezout identity (1) using state-space approach. For example, if we adopt the schemes shown in Fig. 1 and 2, the length of the chained eigenvector will be less.

An explicit formula to find solutions of the polynomial generalized Bezout identity is proposed as follows :
Theorem 2: $\bar{D}(s) \in R[s]^{m \times m}$ and $\bar{N}(s) \in$ $R[s]^{m \times r}$ are left coprime and $\bar{D}(s)$ is assumed to be row reduced. Suppose $m \leq r$ and $\delta_{r_{1}} \bar{N}(s) \leq$ $\delta_{r}, \bar{D}(s)$, the controllable and observable realization of $\bar{D}^{-1}(s) \bar{N}(s)=D_{0}+C(s E-A)^{-1} B_{o}$, remain the same as in Lemma 1 . The following eight polynomial matrices satisfy (1) :

$$
\begin{gathered}
\bar{D}(s)=I+C M^{-1}(s) L \\
\bar{N}(s)=D_{o}+C M^{-1}(s)\left(B_{o}+L D_{0}\right) \\
D(s)=\left(I+K H^{-1}(s) B_{0}\right) \\
N(s)=D_{0}+\left(C+D_{o} K\right) H^{-1}(s) B_{o} \\
\bar{X}(s)=I-\left(C+D_{0} K\right) H^{-1}(s) L \\
\bar{Y}(s)=K H^{-1}(s) L \\
X(s)=I-K M^{-1}(s)\left(B_{0}+L D_{o}\right) \\
Y(s)=K M^{-1}(s) L
\end{gathered}
$$

where $M(s)=s E-I, E$ is found from (10) and $H(s)=s E-A-B_{o} K, K$ is obtained from Theorem 1 which is infinite eigenstructure assignment.

To consider the case of $m>r$ in doubly coprime matrix fraction descriptions, we give the following comments.

Lemma 2 : Suppose that $\bar{D}(s) \in R[s]^{m \times m}$ and $\bar{N}(s) \in R[s]^{m \times r}$ are left coprime. It can be easily shown that as $m>r$ and $\mathcal{X}(s)$ is an arbitrary polynomial matrix, the following results hold:

(i) If $\bar{D}(s)$ and $\bar{N}(s)$ are left coprime then $\bar{D}(s)$ and $\left[\bar{N}(s) \vdots[0]_{m \times(m-r)}\right]$ are left coprime.

(ii) If $\bar{D}(s) \bar{X}(s)+\left[\bar{N}(s) \vdots[0]_{m \times(m-r)}\right]\left[\begin{array}{c}\bar{Y}(s) \\ \mathcal{X}(s)\end{array}\right]=I$ then $\bar{D}(s) \bar{X}(s)+\bar{N}(s) \bar{Y}(s)=I$.

(iii) If $\bar{D}(s)[-N(s): \mathcal{X}(s)]+\left[\bar{N}(s):[0]_{m \times(m-r)}\right]$ $\left[\begin{array}{ll}D(s) & \mathcal{X}(s) \\ \mathcal{X}(s) & \mathcal{X}(s)\end{array}\right]=\left[\begin{array}{ll}0 & 0\end{array}\right]$ then $-\bar{D}(s) N(s)+$ $\bar{N}(s) D(s)=0$.

(iv) If $\left[\begin{array}{c}-Y(s) \\ \mathcal{X}(s)\end{array}\right] \bar{X}(s)+\left[\begin{array}{cc}X(s) & 0 \\ \mathcal{X}(s) & \mathcal{X}(s)\end{array}\right]\left[\begin{array}{c}\bar{Y}(s) \\ \mathcal{X}(s)\end{array}\right]=$ $\left[\begin{array}{l}0 \\ 0\end{array}\right]$ then $-Y(s) \bar{X}(s)+X(s) \bar{Y}(s)=0$.

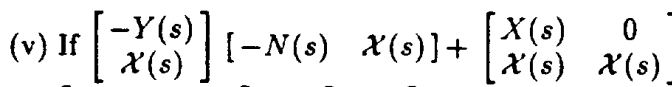
$\left[\begin{array}{ll}D(s) & \mathcal{X}(s) \\ \mathcal{X}(s) & \mathcal{X}(s)\end{array}\right]=\left[\begin{array}{ll}I & 0 \\ 0 & I\end{array}\right]$ then $Y(s) N(s)+$ $X(s) D(s)=1$. 
(vi) If

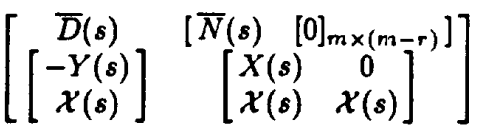

$$
\begin{aligned}
& {\left[\begin{array}{cc}
\bar{X}(s) & {\left[\begin{array}{ll}
-N(s) & \mathcal{X}(s) \\
\bar{Y}(s) \\
\mathcal{X}(s)
\end{array}\right]} \\
{\left[\begin{array}{ll}
D(s) & \mathcal{X}(s) \\
\mathcal{X}(s) & \mathcal{X}(s)
\end{array}\right]}
\end{array}\right]=\left[\begin{array}{ll}
I & 0 \\
0 & I
\end{array}\right]}
\end{aligned}
$$

then

$$
\left[\begin{array}{cc}
\bar{D}(s) & \bar{N}(s) \\
-Y(s) & X(s)
\end{array}\right]\left[\begin{array}{cc}
\bar{X}(s) & -N(s) \\
\bar{Y}(s) & D(s)
\end{array}\right]=\left[\begin{array}{cc}
I & 0 \\
0 & I
\end{array}\right]
$$

(vii) $\bar{D}(s)$ and $\left[\bar{N}(s) \vdots[0]_{m \times(m-r)}\right]$ are left coprime, the polynomial generalized Bezout identity can be written as

$$
\begin{gathered}
{\left[\begin{array}{cc}
\bar{D}(s) & {\left[\bar{N}(s):[0]_{m \times(m-r)}\right]} \\
-Y_{e}(s) & X_{e}(s)
\end{array}\right]} \\
{\left[\begin{array}{cc}
\bar{X}_{e}(s) & -N_{e}(s) \\
\bar{Y}_{e}(s) & D_{e}(s)
\end{array}\right]=\left[\begin{array}{ll}
I & 0 \\
0 & I
\end{array}\right]}
\end{gathered}
$$

The form of $\bar{D}(s),\left[\bar{N}(s):[0]_{m \times(m-r)}\right], \bar{X}_{e}(s)$, $\bar{Y}_{e}(s), D_{e}(s), N_{e}(s), X_{e}(s)$ and $Y_{e}(s)$ are defined as those indicated in Theorem 2.

Following the Algorithm listed below, (16) can be written as (15), where the form of $X_{\epsilon}(s)$ is $\left[\begin{array}{cc}X(s) & 0 \\ \mathcal{X}(s) & \mathcal{X}(s)\end{array}\right]$

Algorithm : The computation algorithm for solving equation (1) is given below.

Step (i): On the basis of the generalized statespace representation, use Lemma 1 to realize $\bar{D}^{-1}(s)\left[\bar{N}(s):[0]_{m \times(m-r)}\right]$. In the realization procedure, set $B_{\text {ohr }} \in R^{m \times m}$ be a full row rank matrix and $\left(B_{o h r}-N_{h r}\right)$ is nonsingular. Obtain $D_{o}=-D_{h r}^{-1}\left(B_{o h r}-N_{h r}\right)$ which will be nonsingular as well. $\left(B_{o}+L D_{o}\right)$ can be read directly from the coefficients of $\left[N(s):[0]_{m \times(m-r)}\right]-D_{o}$.

Step (ii): Select rank 0 infinite eigenvector as $V_{\infty}^{(0)}=$ $\bar{V}_{\infty}^{(0)} D_{0}$ where $\bar{V}_{\infty}^{(0)}$ is the same as (12) and select $f_{\infty j}^{(0)}=\left[[0]_{1 \times r}:\left[\mathcal{Z}_{j}\right]_{1 \times(m-r)}\right]^{t}$ where $r<j \leq m$ and $\mathcal{Z}_{j}$ are the arbitrary constant matrices. Use Theorem 1 for infinite eigenstructure assignment, then apply Theorem 2 and Lemma 2 to obtain the desired solution.
In the follow, we want to prove the special form of $X_{e}(s)$. We have $A \bar{V}_{\infty}^{(0)}=(I-L C) \bar{V}_{\infty}^{(0)}=\bar{V}_{\infty}^{(0)}-L$,

$$
B_{1} \triangleq B_{0}+L D_{0}=B_{0}-A \bar{V}_{\infty}^{(0)} D_{0}+\bar{V}_{\infty}^{(0)} D_{0}
$$

and

$$
B_{1}-\bar{V}_{\infty}^{(0)} D_{o}=\left[\mathcal{Y}_{n \times r}:[0]_{n \times(m-r)}\right],
$$

where $\mathcal{Y}$ is an arbitrary constant matrix. We know $A \bar{V}_{\infty}^{(0)} D_{o}=-\left[\mathcal{Y}_{n \times r}:[0]_{n \times(m-r)}\right]+B_{0}$. Define $F_{\infty}^{(0)} \triangleq\left[f_{\infty 1}^{(0)} \cdots f_{\infty m}^{(0)}\right], \quad V_{\infty}^{(1)} \triangleq\left[v_{\infty 1}^{(1)} \cdots v_{\infty m}^{(1)}\right]$ and $V_{\infty}^{(0)} \triangleq\left[v_{\infty 1}^{(0)} \cdots v_{\infty m}^{(0)}\right]=\bar{V}_{\infty}^{(0)} D_{0}$ then

$$
\begin{aligned}
& {\left[\begin{array}{l}
V_{\infty}^{(1)} \\
F_{\infty}^{(0)}
\end{array}\right]} \\
& =\left[E \vdots B_{0}\right]^{+} A \bar{V}_{\infty}^{(0)} D_{o} \\
& =\left[E \vdots B_{0}\right]^{+}\left\{-\left[\mathcal{Y}_{n \times r}:[0]_{n \times(m-r)}\right]+B_{o}\right\} .
\end{aligned}
$$

Since $\left[E \vdots B_{o}\right]^{+} B_{o}=\left[[0]_{m \times n} \vdots I_{m \times m}\right]^{t}$, we can easily prove that $\operatorname{rank}\left[V_{\infty}^{(1)}\right] \leq r$. We can also choose $K$ for the desired infinite eigenvectors whose chain have structures as $\sum_{i=1}^{r} p_{\infty i}=n-m+r$ and $p_{\infty j}=$ $1(r<j \leq m)$. The vectors $f_{\infty j}^{(0)}(1 \leq j \leq r)$ can be solved by (7) and the values of $f_{\infty j}^{(0)}(r<j \leq m)$ are determined under the requirement of Algorithm step (ii) listed above. From (5), we may proceed to obtain

$$
\begin{aligned}
& K V_{\infty}^{(0)}=K \bar{V}_{\infty}^{(0)} D_{o}=F_{\infty}^{(0)} \\
& =\left[f_{\infty 1}^{(0)} \cdots f_{\infty r}^{(0)} \cdots f_{\infty m}^{(0)}\right] \\
& =\left[\begin{array}{cc}
\mathcal{Z}_{r \times r} & {[0]_{r \times(m-r)}} \\
\mathcal{Z}_{(m-r) \times r} & \mathcal{Z}_{(m-r) \times(m-r)}
\end{array}\right],
\end{aligned}
$$

where $\mathcal{Z}$ are the arbitrary constant matrices. From (13), (15) and $X_{e}(s)=I-K M^{-1}(s)\left(B_{o}+L D_{o}\right)$, since the constant term of $X_{e}(s)$ is

$$
\begin{aligned}
& I+K\left(B_{0}+L D_{0}\right) \\
& =I+K\left\{\left[\mathcal{Y}_{n \times r}:[0]_{n \times(m-r)}\right]+\bar{V}^{(0)} D_{0}\right\} \\
& =I+\left[K \mathcal{Y}_{n \times r}:[0]_{m \times(m-r)}\right]+F_{\infty}^{(0)}
\end{aligned}
$$

and the $s^{i}$ term of $X_{e}(s)$ is

$$
\begin{aligned}
& K E^{i}\left(B_{o}+L D_{o}\right) \\
& =K E^{i}\left\{\left[\mathcal{Y}_{n \times r}:[0]_{n \times(m-r)}\right]+\bar{V}^{(0)} D_{o}\right\} \\
& =\left[K E^{i} \mathcal{Y}_{n \times r}:[0]_{m \times(m-r)}\right], \text { where } 0<i \leq q-1,
\end{aligned}
$$

the resulting form of $X_{e}(s)=I-K M^{-1}(s)\left(B_{0}+\right.$ $\left.L D_{o}\right)$ is $\left[\begin{array}{cc}X(s) & 0 \\ \mathcal{X}(s) & \mathcal{X}(s)\end{array}\right]$. 


\section{Example}

Given

$$
\begin{aligned}
& \bar{D}(s)=\left[\begin{array}{cc}
s^{2}+3 s+2 & 0 \\
s & s^{2}+1
\end{array}\right], \\
& \bar{N}(s)=\left[\begin{array}{cc}
s^{2}+1 & s \\
0 & s^{2}+s+1
\end{array}\right]
\end{aligned}
$$

find the desired eight polynomial matrices $\bar{D}(s)$, $\bar{N}(s), \bar{X}(s), \bar{Y}(s), D(s), N(s), X(s)$, and $Y(s)$ that satisfy the following equality

$$
\left[\begin{array}{cc}
\bar{D}(s) & \bar{N}(s) \\
-Y(s) & X(s)
\end{array}\right]\left[\begin{array}{cc}
\bar{X}(s) & -N(s) \\
\bar{Y}(s) & D(s)
\end{array}\right]=\left[\begin{array}{cc}
I & 0 \\
0 & I
\end{array}\right]
$$

\section{Solution :}

The closed-loop infinite eigenvector chain lengths are $p_{\infty 1}=3$ and $p_{\infty 2}=3$, then

$$
\begin{aligned}
& D(0)=\left[\begin{array}{ll}
-0.111^{2}+0.228-0.22 & 0.580^{2}-0.118+1.11 \\
-0.561^{2}+0.000-0.56 & 1.788^{2}+0.000+1.78
\end{array}\right] . \\
& N(0)=\left[\begin{array}{ll}
-0.118^{2}-0.000-0.11 & +0.560^{2}-0.000+0.36 \\
-0.368^{2}-0.41-0.36 & +1.781^{2}+1.220+1.78
\end{array}\right], \\
& \bar{x}(0)=\left[\begin{array}{ll}
-0.11 r^{2}-0.401+0.08 & 0.568^{2}+0.40 t+0.36 \\
-0.368^{2}-0.841-0.36 & 1.780^{2}+2.620+2.58
\end{array}\right] \text {, } \\
& \bar{V}(0)=\left[\begin{array}{l}
+0.110^{2}+0.180+0.02-0.568^{2}-0.291-0.71 \\
+0.368^{2}+0.400+0.36-1.788^{2}-1.400-1.38
\end{array}\right] \text {. } \\
& x(0)=\left[\begin{array}{ll}
1.0^{2}-4.4 .+3.2 & 0.0^{2}+0.11-7.2 \\
0.0^{2}-1.60+0.8 & 1.0^{2}+160-0.8
\end{array}\right] \text {. } \\
& Y(0)=\left[\begin{array}{cc}
-1 . .^{2}+1.40+0.6 & -0.0^{2}+0.60+2.2 \\
0.0^{2}+0.60+3.4 & -1.0^{2}-0.61-0.2
\end{array}\right]
\end{aligned}
$$

If the infinite eigenvector chain lengths are $\bar{p}_{\infty 1}=$ 5 and $\bar{p}_{\infty 2}=1$, then

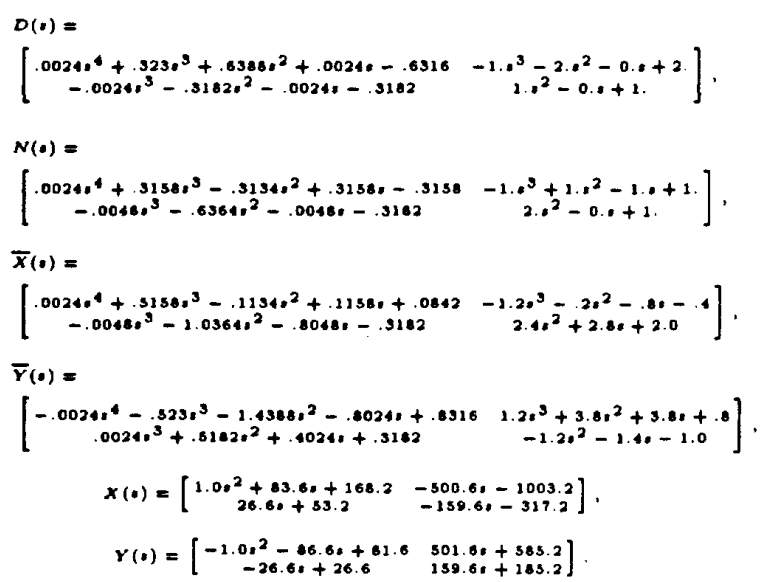

\section{Conclusions}

The major contributions of this note are as follows

(i) A controllable and observable generalized statespace realization method for polynomial matrix fraction descriptions is constructed.

(ii) An efficient algorithm to treat infinite eigenstructure assignment problems is developed.

(iii) To reduce the lengths of closed-loop infinite eigenvector chains, we provide row search sequences as shown in Fig. 1 and Fig. 2.

(iv) Explicit formulas for solving the polynomial generalized Bezout identity are derived. Since the chain lengths of closed-loop infinite eigenvectors are considered during the state feedback gain computation procedures, we can find the adjustable-degree solutions based on the generalized state-space approach.

The developed method allows us to use the existing software packages (e.g. MATRIX $X_{X}$, $M A T L A B$ etc.) to compute the solution.

\section{Reference :}

[1] T. Beelen and P. Van Dooren, "A pencil approach for embedding a polynomial matrix into a unimodular matrix," SIAM J. Matrix Anal. Appl., Vol. 9, pp. 77-89, 1988.

[2] F.M. Callier and C.A. Desoer, Multivariable feedback systems. New York, NY : SpringerVerlag, 1982.

[3] F.R. Chang, C.H. Fang, and C.H. Wang, "Doubly coprime matrix-fraction representations using proportional and derivative feedback concepts in generalized state-space systems," IEEE Trans. Automat. Contr., Vol. 36, pp. 11931195,1991

[4] F.R. Chang, C.H. Wang, C.H. Fang and S.D Wang, "Solving the generalized Bezout identity by a state space approach," Control-Theory and Advanced Tech., Vol. 8, pp. 269-287, 1992.

5] M.M. Fahmy and J.O'Reilly, "Matrix pencil of closed-loop descriptor systems: infiniteeigenvalue assignment," Int. J. Control, Vol. 49, pp. 1421-1431, 1989. 
[6] C.H. Fang, "A new Approach for calculating doubly coprime matrix fraction descriptions," IEEE Trans. Automat. Contr., Vol. 37, pp. 138-141, 1992.

[7] C.H. Fang and F.R. Chang, "A strongly observable and controllable realization of descriptor systems," Control-Theory and and Advanced Tech., Vol. 6, pp. 133-141, 1990.

[8] T. Kailath, Linear systems. Englewood Cliffs, NJ: Prentice-Hall, 1980.

[9] V. Kucera, Discrete linear control, New York, NY : Wiley, 1979.

[10] P. Lancaster and M. Tismenetsky, The theory of matrices, New York, NY : Academic Press, 1985.

[11] F.L. Lewis, "A survey of linear singular systems," Circuits, System, Signal Processing, Vol.5, pp. 3-36, 1986.

[12] R.V. Patel, "Computation of matrix fraction descriptions of linear time-invariant systems, IEEE Trans. Automat. Contr., Vol. 26, pp. 148-161, 1981.

[13] G.C. Verghese, B.C. Levy and T. Kailath, "A generalized state-space for singular systems," IEEE Trans. Automat. Contr., Vol. 26, pp. 811-831, 1981.

This work was supported in part by the National Science Council of the Republic of China under grant number NSC80-0404-E002-52 